\title{
Manger à Pâques en Grèce égéenne
}

Résidence uxori-matrilocale et chevreau farci

Eating at Easter in Aegean Greece: Uxori-matrilocal Residence and Stuffed Kid

\section{Katerina Melissinou}

\section{CpenEdition}

Journals

Édition électronique

URL : https://journals.openedition.org/ceb/7135

DOI : $10.4000 /$ ceb. 7135

ISSN : 2261-4184

Éditeur

INALCO

Édition imprimée

ISBN : 978-2-85831-230-6

ISSN : 0290-7402

Référence électronique

Katerina Melissinou, « Manger à Pâques en Grèce égéenne », Cahiers balkaniques [En ligne], Hors-série 2016, mis en ligne le 16 mars 2017, consulté le 06 juillet 2021. URL : http://journals.openedition.org/ ceb/7135; DOI : https://doi.org/10.4000/ceb.7135

Ce document a été généré automatiquement le 6 juillet 2021.

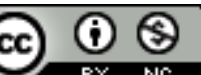

Cahiers balkaniques est mis à disposition selon les termes de la Licence Creative Commons Attribution - Pas d'Utilisation Commerciale 4.0 International. 


\title{
Manger à Pâques en Grèce égéenne
}

\author{
Résidence uxori-matrilocale et chevreau farci \\ Eating at Easter in Aegean Greece: Uxori-matrilocal Residence and Stuffed Kid
}

Katerina Melissinou

1 Ma contribution consiste à mettre en œuvre les rapports entre cuisine rituelle de Pâques en Grèce contemporaine et représentations du genre, notamment à travers le mode traditionnel de résidence post-marital, c'est-à-dire le lieu d'habitation du jeune couple, dans le sillage du travail de Nicole-Claude Mathieu sur la construction de la personne femme en sociétés uxorilocales (2007).

2 Mode de résidence, souvent évocateur du système de parenté, et manières de table dans une société «étudiée » comme disent les anthropologues, ont été un des jeux préférés du structuralisme de Lévi-Strauss dans les années 1970. Dans mon exemple, je vais traiter de deux pratiques de résidence et de deux types d'accommodation du plat pascal, qui matérialisent les représentations du genre et du corps procréateur sur le mode de la production de l'espace et sur le mode culinaire.

3 En matière de résidence post-maritale on pourrait distinguer, en généralisant, deux pratiques en Grèce. En Grèce continentale, y compris les îles de la mer Ionienne ainsi qu'en Crète, la résidence post-maritale est au moins jusqu'aux années 1960 viripatrilocale, le jeune couple habitant chez le marié (virilocale), et souvent chez le père du marié (viri-patrilocale). Ce type de résidence est remplacé aujourd'hui par des pratiques néolocales des couples, en principe entraînées par l'exode rural de l'aprèsguerre.

4 Or en Grèce égéenne, c'est-à-dire dans les îles depuis Lemnos au nord-est jusqu'à Andros à l'ouest et Kastellorizo au sud-est, les pratiques d'habiter post-maritales se trouvent aux antipodes de celles déjà mentionnées. Dans la terminologie de l'anthropologie (COLLECTIF, 2000 ; COLleCTIF, 2007) ce type de résidence s'appelle uxorimatrilocale, c'est-à-dire chez l'épouse (uxorilocale), et souvent chez la mère de l'épouse (uxori-matrilocale). Je définis comme traditionnel le système de résidence avant l'exode rural de l'après-guerre, bien que, en dehors des cas de résidence néolocale, les 
pratiques de résidence post-maritale n'aient pas changé et encore moins celles de la dévolution des maisons à la génération suivante.

Ces pratiques de résidence post-maritale créent forcément des représentations de l'espace différentes dans les deux systèmes. Alki Kyriakidou-Nestoros (1979), étudiante de Lévi-Strauss, et après elle Eleni Tzavara (1986), ont démontré comment la pratique continentale laisse son empreinte dans des fêtes locales, dont le repas de Pâques, qui se passe à l'extérieur de la maison et qui réunit des réseaux de parenté liés à travers les hommes, des hommes qui tournent chacun à leur tour des agneaux passés à la broche.

En effet le plat pascal pour la Grèce traditionnellement patri-virilocale consiste dans le fameux agneau rôti à la broche, accommodé par des hommes, coutume qui tire son origine des klephtes de Roumélie du $\mathrm{xIx}^{\mathrm{e}}$ siècle, mais il s'agit d'une manière très répandue qui homogénéise dans l'imaginaire national et touristique une pratique, à l'origine, localisée dans la Roumélie.

7 Avant de quitter l'agneau à la broche des hommes, évoquons aussi la pratique de l'agneau rôti au four, souvent faute d'espace pour la broche, mais aussi relevant d'autres modes d'occupation de l'espace et de pratiques d'habitation, en dehors d'une dichotomie généralisante patri/matri. Je pense au Péloponnèse surtout où le système de dévolution des maisons et des noms échappe à la dichotomie sus-mentionnée (COUROUCLI, 1987).

8 Mais alors comment le préparent-ils dans les îles? La question doit être formulée différemment. Comment le préparent-elles dans les îles? Ici l'optique change radicalement. Ce sont les maitresses de maison qui sont impliquées, le repas se passe à l'intérieur des maisons des îles, de petites maisons, comme on le sait, dans des cuisines à l'abri du soleil, et les convives sont des membres de la famille nucléaire ; s'il y a des invités, il s'agit des parents matrilatéraux, c'est-à-dire de la parenté du côté de la mère. Le plat pascal consiste en du chevreau plutôt qu'en de l'agneau au four, comme dans d'autres parties de Grèce d'ailleurs (comme le Péloponnèse ou la Thessalie), avec une différence majeure : ici, la carcasse est farcie.

\section{D'une matérialité à l'autre : espace et cuisine}

9 Le plat pascal des îles uxori-matrilocaux consiste donc en chevreau farci au four. J'ai constaté que dans les îles des Cyclades et de l'égée orientale, zone traditionnelle de la résidence matri-uxorilocale, cette recette pascale est de rigueur. Partout? Non. Pas dans des communautés arvanites ou catholiques d'Andros, de Tinos ou de Syros. Le mode de transmission des maisons dans ces communautés corrobore mon hypothèse du rapport entre l'uxori-matrilocalité et la carcasse farcie, puisque, dans ces communautés où le rôti pascal n'est pas farci, les maisons ne se transmettent pas de mère en fille. Cette pratique post-maritale, qui attribue au couple comme espace conjugal l'espace féminin, n'en dit-elle pas davantage sur l'idéologie du genre et sur le corps procréateur féminin, à travers non pas une, mais deux matérialités, la maison natale d'une part maternelle, dans laquelle se perpétueront les naissances d'héritières filles-aînées, et d'autre part la matière culinaire du chevreau farci ? Lévi-Strauss a lié ce type médiatisé de cuisson avec des sociétés à inflexion matrilinéaire pour ce qui est du système de parenté, ou simplement uxori-matrilocales. Son fameux triangle culinaire, inspiré du triangle des voyelles de Saussure, démontrait l'opposition entre la cuisson au contact 
au feu et la cuisson médiatisée soit par un récipient, soit par un récipient et un liquide dans lequel cuisait la substance alimentaire $(1990,21)$.

10 J'ai effectué mon travail de terrain de thèse dans un village uxori-matrilocal, Apíranthos, situé à Naxos, une île des Cyclades. Dans ce village, pour le chevreau farci, la carcasse est vidée de ses viscères pour être remplie d'herbes, de riz et de raisins secs. Dans d'autres villages et dans d'autres îles on trouve des versions différentes de cette farce, où le riz est remplacé par de la mie de pain, ou du blé concassé, quelques recettes incluant de la viande hachée ou des viscères coupés fin (BARBARIGOU, 2002 ; GRIGORIADOU, 2003 ; ORGANISME de tourisme du Dodécanèse, 2003 ; VAGIAKOU, 2000 ; YERASIMOU, 2004), mais ce qui reste invariable dans la farce, comme une sorte de constante, c'est la participation d'herbes aromatiques de printemps combinées aux céréales : la menthe et le persil, cultivés en pots dans la cour, le máratho, feuilles de fenouil sauvage, et l'aneth, qui poussent dans les jardins au printemps, viennent parfumer la farce. La farce s'appelle dans le village étudié pátoudha ${ }^{1}$, (un neutre pluriel) et elle donne son nom au plat pascal entier'2. C'est ainsi que, par métonymie, le plat n'étant plus la viande mais sa farce, le plat-farce est pensé comme doublement médiatisé s'agissant de son mode de cuisson : cuit dans la viande, puis la viande cuite dans le plat au four, il se trouve à l'opposé du rôti à la broche, par rapport à son contact au feu, comme le bouilli qui est médiatisé par le récipient et l'eau dans laquelle il est immergé (LÉVI-STRAUSS, art. cit.). Dans la recette d'Apíranthos, les herbes consistent en blettes, séfla, et tiges tendres, koutsounádhes, du coquelicot, d'une part, et en aromates tels l'aneth, anitho et le fenouil sauvage, máratho, de l'autre. Tout l'axe du fade au parfumé - j'y reviendrai - est sollicité et se retrouve à l'intérieur de la viande, car ce qui prévaut dans le discours autour des pátoudha, c'est l'odeur. Devant une farce réussie on parle de pátoudha "myrodháta", «odorants, parfumés ». L'odeur serait-elle la quintessence d'une substance interne? Comme le disent Manuela Ivone Cunha et Jean-Yves Durand, "la vue s'arrête à la surface, aux apparences» alors qu'« une odeur peut être une "essence": l'odorat ouvrirait [...] l'accès à la nature profonde de l'être et des choses, à leur intériorité » (1999, 172-173).

11 Traditionnellement la viande farcie subissait une cuisson lente dans le four commun où les femmes du quartier faisaient habituellement cuire le pain, et la cuisson des agneaux commençait dès le Samedi saint après la fin de la cuisson du pain et durait jusqu'au dimanche de Pâques, tard dans l'après-midi pour les derniers plats au four. Avec la disparition des fours collectifs dans les quartiers du village, le plat pascal est toujours cuit lentement dès le dimanche matin très tôt et tous les foyers du village passeront à table dans le courant ou même à la fin de l'après-midi. Une cuisson lente au sein de laquelle une autre est comprise : celle de la farce.

\section{La farce, les « Jardins d'Adonis », le fade et le parfumé}

La farce, faite d'herbes fades et d'aromates, de riz et de raisins secs, est d'abord blanchie avec de l'huile d'olive dans une marmite. La cuisson sera parachevée « dans le khonári »3, " tube digestif », du chevreau au four. C'est dans le khonári de la bête, lieu de coction de la nourriture de son vivant, mais aussi lieu des viscères sanguins, que se fera cette coction/digestion des pátoudha qui sont réputés pour donner leur odeur à la viande. La cuisson de la farce à l'intérieur du tube digestif allie la transformation naturelle de la digestion au sein du corps avec celle de la cuisson médiatisée par le feu 
alimentaire. Une fois la farce sortie, des exclamations fusent de tous côtés, car, comme on nous l'a dit, « Pâques, c'est les pátoudha ».

Le moment est venu d'examiner de plus près les ingrédients de cette farce. Comme le disent les gens, il s'agit « d'herbes fades et d'aromates ». Dans la rubrique du fade sont classées la blette et les tiges tendres du coquelicot, dans celle des aromates, l'aneth et le fenouil. Le riz, représentant les céréales, ailleurs dans les îles égéennes remplacé par de la mie de pain ou du blé concassé, et les raisins mûris et séchés sous le soleil, évoquent la «mythologie-cadre» dont parle Jean-Pierre Vernant, contexte dans lequel se positionnent les Jardins d'Adonis, tels que Marcel Detienne les analyse dans sa Mythologie des aromates $(1989,22)$. Or, si les jardins d'Adonis sont présents en Grèce aujourd'hui sous forme de petits pots germés comme le signale un article portant sur la ville de Serrès en Macédoine grecque orientale (PILITSIS, 1985), ce n'est que pendant la Semaine sainte, et notamment le jour de la mort du Christ, le Vendredi saint. Ces «jardins » consistent en céréales poussées dans des petits pots dérisoires, expressément destinées à faner. Dans le cas qui nous occupe ici, le riz et les aromates, qui seraient l'équivalent des jardins d'Adonis d'aujourd'hui ou d'antan, jouent le premier rôle sur la table pascale et ne sont pas liés aux rituels de la mort. Ils sont aussi présents dans le discours comme préoccupation principale de toutes les femmes dont les conversations tournent autour de leur approvisionnement dans les champs aux abords du village. Leur cueillette renforce les réseaux féminins tout en restituant l'espace aux alentours du village aux femmes qui sortent à leur recherche.

Aussi bien dans le rituel des Adonies de la Grèce antique qu'en Grèce du Nord où sont présents les jardins d'Adonis au-delà de la ressemblance des ingrédients des pots que j'ai repérée entre les cas susmentionnés et la farce pascale des îles, il me semble non moins fécond pour la suite de mon analyse de dresser la liste des oppositions. Dans la Grèce antique, les femmes athéniennes montaient les petits pots germés sur le toit de la maison (celle de leur mari bien entendu), afin que, exposés au soleil, ils se fanent. En Grèce du Nord, aujourd'hui, les femmes pleurent avec leurs jardins fanés la mort du Christ le Vendredi saint. Il s'agit de deux cas de pratique viri-patrilocale post-maritale. À l'inverse, dans le cas naxiote, les femmes remplissent de cette farce la viande festive en l'enfonçant doublement dans leur maison à elles: dans le khonári de l'agneau d'abord et ensuite dans le four électrique de la cuisine au rez-de-chaussée de la maison, ou bien chez le boulanger, après l'abandon des fours à bois de la cour. L'opposition ne se réduit pas à la catégorie spatiale extérieur/intérieur, ou virilocale/uxorilocale; c'est la finalité des «jardins » qui varie dans ces trois cas. Les Adonies de la Grèce antique pleuraient la mort d'Adonis comme le font aujourd'hui les femmes de la Grèce du Nord qui offrent leurs miniatures de jardins au tombeau du Christ. La farce pascale des îles est par contre préparée après la " première Résurrection ", qui a lieu le Samedi saint au matin et c'est sa (ré)-apparition de l'intérieur de la viande, et non sa disparition, qui constitue le point culminant de Pâques.

Voyons de près l'ingrédient de la farce le plus rare dans la cuisine locale : les pousses du coquelicot, les koutsounádhes. La légende qui accompagne en Grèce la mort du Christ indique que les gouttes du sang qu'il a versées ont fait jaillir de terre des anémones ou des coquelicots. Effectivement, c'est le seul cas dans la pratique culinaire du village où l'on voit les pousses du coquelicot et les blettes jouer un rôle. Pour ce qui est du fenouil, Marcel Detienne le place au plus près des substances parfumées, en tous cas aux antipodes de la laitue par exemple qui, comme les blettes de notre recette, est 
considérée comme une plante aqueuse, fade, évocatrice de la pourriture et de la mort. Il s'agit donc d'allier des éléments inodores et des éléments parfumés par le mélange d'herbes fades et d'herbes parfumées. Or bien que fades, les pousses du coquelicot jouissent d'un traitement tout différent des blettes dans le discours de ces journées. Comme les blettes sont achetées, leur approvisionnement ne pose pas de problème. Ce sont les koutsounádhes par contre qui font l'objet de discussions et de stratégies d'approvisionnement tant dans le village où on les trouve dans les champs que - et surtout - à Athènes, où on les achète dans quelques rares marchés pendant une assez courte période, et qui envahissent les conversations téléphoniques. Les pousses du coquelicot, bien que fades comme on l'a vu, sont donc chargées d'une valeur symbolique différente des blettes et assez considérable.

16 Le résultat de la farce comme mélange est le suivant : non seulement les pátoudha sont réputés pour leur parfum, mais c'est précisément ce parfum qui l'emporte sur celui de la viande. La viande devient parfumée grâce aux pátoudha, d'autant plus que le parfum émane de la place des viscères sanguins qui ont été enlevés, morceaux de la carcasse les plus aptes à pourrir. Ainsi se produit une sublimation dirait-on, du lieu sanguin, du lieu de la transformation des aliments par la digestion, en un lieu productif de parfum, signe d'intériorité (MELISSINOU, 2015).

\section{Le fil rouge des enjeux du lexique}

17 Le terme qui désigne la tige du coquelicot, koutsounádha, renvoie à un autre terme vernaculaire : koutsoúna. La koutsoúna est une sorte de poupée confectionnée pour et par les petites filles avec des chiffons. Mais koutsoúna est aussi le terme qui désigne ces brioches de Pâques, les tsourekia, en forme de tresse et décorées d'un œuf teint en rouge, que les marraines offrent à leurs filleules comme cadeau de Pâques tout au long de l'enfance. Les koutsoúnes sont donc des poupées ou des brioches, propres à l'âge enfantin ${ }^{4}$. À la puberté, les marraines cessent d'offrir ces brioches à leurs filleules, qui ont elles-mêmes cessé de jouer avec les koutsoúnes (poupées) improvisées. Le mot koúkla, le terme du grec standard pour " poupée », le signifié, remplacera désormais son signifiant koutsoúna, mais maintenant pour désigner autre chose que la poupée: l'écheveau de fil de coton que les femmes utilisent pour confectionner les dentelles et qui est vendu sous forme de tresse, enroulé comme les brioches de Pâques. Toutefois, le terme koúkla désigne aussi bien les poupées des années soixante, dont la présence sur le canapé de la maison était courante jusque dans les années quatre-vingt. Les femmes enceintes saisissaient ces poupées -presque toujours en robe de mariée- lorsqu'elles sentaient le fotus bouger. Par ce geste, elles garantissaient la beauté du bébé.

Il semble donc que la koutsoúna, poupée ou brioche, la koúkla, poupée trônant sur le canapé ou écheveau de coton ou de laine, et la koutsounádha, tige tendre du coquelicot, soient liées toutes les trois à des moments différents des âges d'une femme. La première, poupée ou brioche évoque l'enfance, la seconde, poupée en robe de mariée sur le canapé, est liée à un moment particulier de la grossesse tandis que le fil de coton ou de laine sert à confectionner des dentelles ou des tissus tissés au métier, très valorisées dans les trousseaux féminins. La troisième étape dans cet itinéraire lexical, la koutsounádha, est dissimulée à l'intérieur du chevreau par les cuisinières, femmes mariées. 
Enfin, dans un lapsus culinaire dirait-on, nous trouvons à l'intérieur du chevreau ce qui est normalement à l'extérieur, du fil, de la laine pour ainsi dire. Ce qui vient comme koutsounádha, le remplissage/la farce du chevreau, est en fait par la chaîne de glissement étymologique et sémantique ce qui enveloppe le corps, à l'extérieur, le dérivé culturel de la laine, du fil. Mais la koutsoúna et la koutsounádha ont quelque chose de rouge, puisqu'elles dérivent (j'y reviendrai) du terme kokkinoúdha, la rouge.

Tous ces fils qui créent des entités extérieures renvoient dans le contexte étudié à l'activité interne du corps féminin. "L'intérieur du corps de la femme", nous dit Hippocrate dans son livre Des glandes, "est comme de la laine ", dans un jeu entre l'intérieur et l'extérieur du corps féminin (cité par ANGELOPOULOU A., 1989).

Dans un dialecte bulgare, dit Georges Drettas, le métier à tisser est dit mauka, mère (1980). Comme la vache mère de Cendrillon, dans la version chinoise du conte de fées commentée par Muriel Djéribi (1989) et dans la version yougoslave analysée par Anna Angelopoulou (2004), où l'exploit exigé de Cendrillon par ses sœurs est de filer d'énormes quantités de laine : la pauvre fille est aidée par sa mère transformée en vache. Comment celle-ci l'aide-t-elle? Elle mâche la laine, puis la fait sortir par son oreille sous forme de fil.

2 Toutefois, ce qui renforce l'idée sous-jacente de la physiologie féminine et des fonctions procréatrices des femmes à travers l'usage de la koutsounádha dans le plat pascal d'Apíranthos est son origine étymologique kokkinoúdha, « la rouge », dont koutsounádha est un dérivé. Il faut noter ici qu'un des motifs du métier à tisser, brodé en fil rouge, s'appelle l'ombilic, dans un jeu d'alternance sémantique et symbolique entre fil/cordon ombilical, manié par la tisserande, et ombilic sur le tissu/corps confectionné.

À Santorin, île voisine, les boutons du coquelicot $^{5}$ sont désignés par le terme « artichaut ", terme qui, dans le contexte naxiote, désigne le sexe féminin. C'est le sexe féminin en rouge qui est l'image à laquelle renvoie l'emploi de l'herbe emblématique du plat pascal. Un troisième élément, qui nous incite à penser à la vie intra-utérine, association incontournable dans le contexte de la carcasse farcie et de l'admiration que suscite la sortie de la farce, est l'emploi par la médecine du terme grec ancien, mécon, " coquelicot », pour désigner les premiers excréments intra-utérins du foetus du terme : méconium. Ainsi, sous cet angle étymologique, ce sont aussi les substances intrautérines qui sont fêtées à l'intérieur de la carcasse.

donc toutes ces représentations des âges des femmes et du corps féminin, symboles de génération et de régénération, dans un chevreau qui de plus est toujours mâle ? Les interviews que j'ai faites auprès de femmes et d'hommes, sur place et même à Athènes dans les années 1990-2000, ont dévoilé le substrat ethno-biomédical corporel de la recette en question. L'idée savante de l'ovulation n'existant pas, c'est au contraire la menstruation, le summum de la fécondité des femmes, c'est donc le sang menstruel et matriciel qui fabrique l'enfant.

Les pousses du coquelicot, tellement chargés symboliquement dans ce plat pascal, trouvent ainsi leur place dans un réseau de sens et de sensibilités, de théories sur la fonction du corps procréateur et de pratiques de l'espace.

À la fin de ce parcours nous voyons, comme André-Georges Haudricourt le souligne, "comment un vocabulaire technique», en l'occurrence une pratique culinaire, «constitue un témoignage collectif et inconscient plus sûr et plus objectif que le témoignage explicite et conscient » $(1987,160)$. Dans le cas étudié ici, une pratique et 
un vocabulaire culinaires, collectifs et inconscients révèlent l'idéologie du genre et les représentations du corps sexué, à travers l'étude des mots, des sens et des sensibilités locales de la matière.

\section{BIBLIOGRAPHIE}

ANGelopoulou Anna, 1989, «Fuseau des Cendres », Cahiers de littérature orale, $\mathrm{n}^{\circ}$ 25, p. 71-94.

ANGELoPoulou Anna, 2004, Ellinika Paramythia 2 [Contes grecs II], Athènes : Estia.

BARBARIGOU Argyro, 2002, Syntagès Aigaiou [Recettes de l'Égée], Athènes : Livani.

COLLECTIF, 2000, « Glossaire de la Parenté », l’Homme 154-155, p. 721-732.

COLLECTIF, 2007, « Glossaire », in MATHIEU Nicole-Claude, Une maison sans fille est une maison morte. La personne et le genre en société matrilinéaires et/ou uxorilocales, Paris : MSH, p. 493-503.

COUROUCLI Maria, 1987, « Dot et société en Grèce moderne » in RAVIS-GIORDANI (dir.) Femmes et patrimoine dans les sociétés rurales de l'Europe méditerranéenne, Paris : CNRS, p. 327-348.

CUNHA Manuela-Ivonne \& DURAND Jean-Yves, 1999, « Odeurs, odorats, olfaction : une ethnographie osmologique ", in Danielle MUSSET \& FABRE-VASSAS Claudine, (textes réunis et publiés par), odeurs et parfums, Congrès national des sociétés historiques et scientifiques, 121e, Nice, 26-31 oct. 1996, Paris : CTHS, p. 161-177.

DRETTAS Georges, 1980, la Mère et l'outil. Contribution à l'étude sémantique du tissage rural dans la Bulgarie actuelle, Paris : Société d'Études Linguistiques et Anthropologiques de France, SELAF, « Langues et Civilisations à Tradition Orale, $\mathrm{n}^{\circ} 38$ ».

DODEKANISSIAKOS ORGANISMOS TOURISMOU [Organisme de tourisme du Dodécanèse), 2003, Gefseis Dodekanissou [Saveurs du Dodécanèse], Athènes : Topio.

FABRE-VASSAS Claudine, 1994, la Bête singulière. Les juifs, les chrétiens et le cochon, Paris : Gallimard.

GRIGORIADOU Efi, 2003, Edhesmatologion Smyrnis [Répertoire de mets de Smyrne], Athènes : Kochlias. HAUDRICOURT André-Georges \& DIBIE Pascal, 1987, les Pieds sur terre, Paris : Métailié.

KYRIAKIDOU-NESTOROS Alki, 1979, [1973], «Ta ellinika hristougenniatika fagita kai o symvolismos tous» [« Les mets grecs de Noël et leur symbolisme »], in KYRIAKIDOU-NESTOROS Alki (dir.), Laographika meletimata [Études de folklore], Athènes : Nea Synora Livani.

LAMBRAKI Myrsini, 1997, Ta horta [les Herbes], Athènes : Ellinika Grammata.

LÉVI-STRAUSS Claude, 1990, « Le triangle culinaire », l'Arc, Paris : Duponchelle, p. 19-29.

MATHIEU Nicole-Claude (dir.), 2007, Une maison sans fille est une maison morte. La personne et le genre en sociétés matrilinéaires et/ou uxorilocales. Paris : Maison des sciences de l'homme.

MELISSINOU Katerina, 2015, « Sens et sensibilités de l'intériorité. Espace, cuisines et corps : essai d'écrire les sens», Traverse, Par tous les sens, $n^{\circ} 2$, p. 119-129. 
PILITSIS Georges, 1985, “The gardens of Adonis in Serres today”, Journal of Modern Greek Studies, Volume 3, no 2, October 1985, pp. 145-166.

TZAVARA Eleni, 1986, «I mageiriki ston elliniko agrotiko horo. Stoiheia analysis tou systimatos» [« La cuisine dans le milieu agricole grec. Des éléments pour une analyse du système »], in LAGOPOUlOS A.-Ph., MARTINIDIS P., BOKLUND-LAGOPOULOU C. \& SPYRIDONIDIS C.-V. (dir.), I dynamiki ton simeion. Pedia kai methodoi mias koinoniosimeiotikis [la Dynamique des signes. Des champs et des méthodes pour une socio-sémiotique], Salonique : Paratiritis, p. 411-432.

YERASIMOU Marianna, 2004, I Othomaniki Mageiriki [la Cuisine ottomane], Athènes : Potamòs.

VAGIAKOU Ourania, 2000, Syntagès limniakis kouzinas [Recettes de cuisine lémnienne], Athènes :

Limnos.

VERNANT Jean-Pierre, 1989 [1972], « Introduction » in DETIENNE Marcel, les Jardins d'Adonis : la

mythologie des aromates en Grèce, Paris : Gallimard.

\section{NOTES}

1. À côté de ma propre recherche de terrain dans quelques îles des Cyclades et de l'Égée orientale concernant le plat pascal, il existe aussi une bibliographie culinaire récente dans laquelle j'ai trouvé ces versions différentes du plat. Outre les versions différentes du plat que nous fournit la bibliographie, nous pouvons supposer qu'il vient de la côte orientale de la mer Égée, de tradition uxorilocale alors que, selon le dictionnaire de l'Académie d'Athènes, son étymologie trahit une tradition vénitienne: pátoudha<battudo, ce qui est battu ou pétri.

2. Le même terme, ou presque, désigne un plat qui est présent sur les tables de l'île voisine de Paros, à deux différences près : l'époque de la consommation et l'animal ; c'est à Noël que l'on mange à Paros le lièvre pàtoudho: raisins secs, mie de pain, noix, pomme fruit, poivre, cognac, fromage, huile d'olive, citron.

3. Khonári désigne dans le village le tube digestif. Khonévo est, en effet, le verbe qui signifie « digérer ».

4. Il convient de citer ici un parallèle ethnographique en France, développé par Claudine Fabre-Vassas dans son ouvrage la Bête singulière, où les coquelicots sont entre les mains des enfants transformés en enfants de chœur, poupées minuscules, avec leurs pétales retournés vers le bas, formant comme des robes rouges $(1994,65)$.

5. Sur la version kokkinoúdha ainsi que sur le rapprochement entre l'artichaut et les boutons du coquelicot - car à Santorin le coquelicot est appelé aguinarohorto, herbe d'artichaut - j'ai consulté le livre de LAMBRAKI Myrsini (1997, 141). 


\section{RÉSUMÉS}

Sont ici examinés les rapports entre cuisine rituelle de Pâques en Grèce contemporaine et représentations du genre, notamment à travers le mode traditionnel de résidence post-maritale, c'est-à-dire le lieu d'habitation du jeune couple. Ces trois matérialités, la nourriture, le corps et l'espace, font écho à l'idéologie du genre. En Grèce continentale traditionnelle, la résidence postmaritale est patri-virilocale et l'agneau pascal rôti à la broche constitue un moment de célébration des réseaux de parenté agnatique. En Grèce égéenne, en revanche, la résidence postmaritale est matri-uxorilocale. Le plat emblématique pascal des îles de la mer Égée, le chevreau farci, se révèle être lié aux symboles des âges des filles et des femmes, tout en étant foncièrement lié à l'espace produit par des pratiques de résidence. Cuisine et espace sont tous les deux évocateurs des représentations sur le corps et notamment sur le corps procréateur, féminin ou masculin.

This contribution aims at implementing the relationships between Easter ritual cooking in Greece and contemporary representations of gender, among others through the traditional way of post-marital residence, that is to say, the place of residence of the young pair. Those three materialities, food, body and space, do echo gender ideology. In mainland Greece, traditional post-marital residence is patri-virilocal and the Easter lamb, roasted on a spit, is a time of celebration of agnatic kinship networks. Conversely, Aegean Greece post-marital residence is matri-uxorilocal. The emblematic Easter dish of the Aegean islands, stuffed kid, appears to be linked to girls' and women's ages symbols while being also fundamentally linked to the space resulting of dwelling practices. Cooking and space are both evocative of representations of the body-including the reproductive body, male or female.

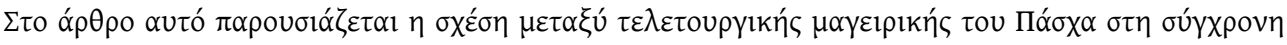

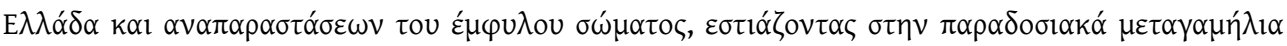

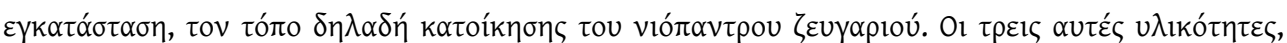

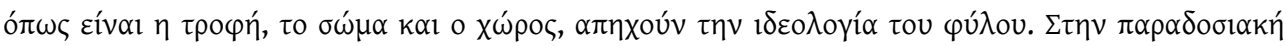

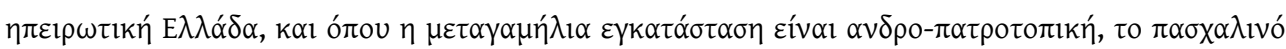

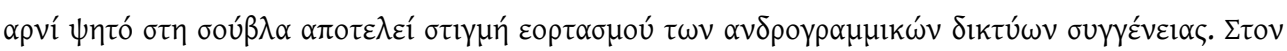

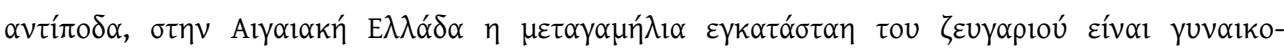

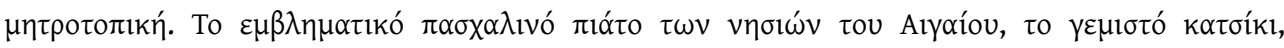

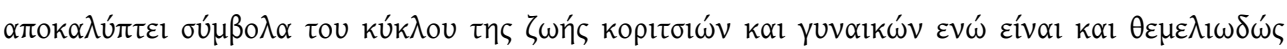

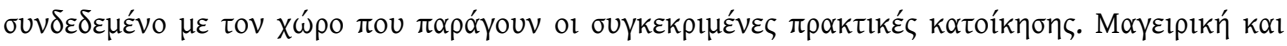

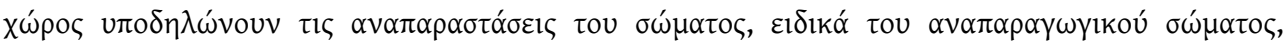

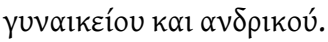


INDEX

Index géographique : Grèce, Égée

Index chronologique : vingt-et-unième siècle

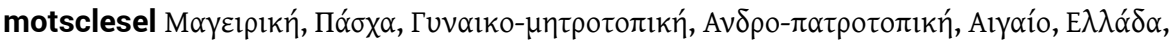

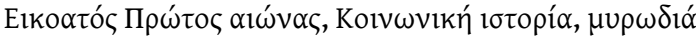

motsclestr Gastronomi, Paskalya, Ege, Yunanistan, Yirmi birinci yüzyıl, Sosyal Tarih

Mots-clés : cuisine, cuisine, pâques, pâques, uxori-matrilocal, uxori-matrilocal, viri-patrilocal, viri-patrilocal, odeur, odeur, parfum, parfum

motsclesmk ГАСТРОНОМИЈА, ВЕЛИГДЕН, ЕГЕЈСКА, ГРЦИЈА, ДВАЕСЕТ И ПРВИОТ ВЕК, СОЦИЈАЛНАТА ИСТОРИЈА

Keywords : Cooking, Easter, Uxori-matrilocal, Viri-patrilocal, Aegean, Greece, Twenty First century, Social history, Smell

Thèmes : Histoire sociale

\section{AUTEUR}

\section{KATERINA MELISSINOU}

EHESS

Université Nationale et Capodistrienne d'Athènes 